\title{
Synthesizing Concurrent Programs using Answer Set Programming
}

\author{
Emanuele De Angelis ${ }^{1}$, Alberto Pettorossi ${ }^{2}$, and Maurizio Proietti ${ }^{3}$ \\ 1 Dipartimento di Scienze, University 'G. D'Annunzio', Pescara, Italy \\ deangelis@sci.unich.it \\ 2 DISP, University of Rome Tor Vergata, Italy \\ pettorossi@disp.uniroma2.it \\ 3 CNR-IASI, Rome, Italy \\ proietti@iasi.cnr.it
}

\begin{abstract}
We address the problem of the automatic synthesis of concurrent programs within a framework based on Answer Set Programming (ASP). The concurrent program to be synthesized is specified by providing both the behavioural and the structural properties it should satisfy. Behavioural properties, such as safety and liveness properties, are specified by using formulas of the Computation Tree Logic, which are encoded as a logic program. Structural properties, such as the symmetry of processes, are also encoded as a logic program. Then, the program which is the union of these two encodings, is given as input to an ASP system which returns as output a set of answer sets. Finally, each answer set is decoded into a synthesized program that, by construction, satisfies the desired behavioural and structural properties.
\end{abstract}

\section{Introduction}

We consider concurrent programs consisting of finite sets of processes which interact with each other through communication protocols. Such protocols are based on a set of instructions, called synchronization instructions, operating on shared variables ranging over finite domains. The communication protocols are realized in a distributed manner, that is, every process includes one or more regions of code consisting of synchronization instructions, responsible for the interaction between processes.

Even for a small number of processes, communication protocols which guarantee a desired behaviour of the concurrent programs may be hard to design. In this paper we propose a method for automatically synthesizing correct concurrent programs starting from the formal specification of their desired behaviour.

Methods for the automatic synthesis of concurrent programs from temporal logic specifications have been proposed in the past by Clarke and Emerson [6], Manna and Wolper [16], and Attie and Emerson [1,2]. All these authors reduce the task of synthesizing a concurrent program to the task of synthesizing the synchronization instructions of each process. We follow their approach and everything which is irrelevant to the synchronization among processes, is abstracted away and each process is considered to be a finite state automaton. 
We introduce a framework, based on logic programming, for the automatic synthesis of concurrent programs. We assume that the behavioural properties of the concurrent programs, such as safety and liveness properties, are specified by using formulas of the Computation Tree Logic (CTL for short), which is a very popular propositional temporal logic over branching time structures (see, for instance, $[5,6])$. This temporal, behavioural specification $\varphi$ is encoded as a set $\Pi_{\varphi}$ of clauses. We also assume that the processes to be synthesized satisfy suitable structural properties, such as a symmetry property, and that those properties can be encoded as a set $\Pi_{\Sigma}$ of clauses. Structural properties cannot be easily specified by using CTL formulas and we use, instead, a simple algebraic structure that we will present in the paper. Thus, the specification of a concurrent program to be synthesized consists of a logic program $\Pi=\Pi_{\varphi} \cup \Pi_{\Sigma}$ which encodes both the behavioural and the structural properties that the concurrent program should satisfy.

We show that every answer set (that is, every stable model) of the program $\Pi$ represents a concurrent program satisfying the given specification. Thus, by using an Answer Set Programming (ASP) system, such as DLV [9] or smodels [20], which computes the answer sets of logic programs, we can synthesize concurrent programs which enjoy some desired properties.

We have performed some synthesis experiments and, in particular, we have synthesized some mutual exclusion protocols which are guaranteed to enjoy various properties, such as (i) bounded overtaking, (ii) absence of starvation, and (iii) maximal reactivity (their formal definition will be given in the paper). We finally compare our results with those presented in $[1,2,12]$.

The paper is structured as follows. In Section 2 we recall some preliminary notions and terminology. In Section 3 we present our framework for synthesizing concurrent programs and we define the notion of a symmetric concurrent program. In Section 4 we describe our synthesis procedure and the logic program which we use for the synthesis. In Section 5 we present some examples of synthesis of symmetric concurrent programs. Finally, in Section 6 we discuss the related work and some topics that can be investigated in the future.

\section{Preliminaries}

Let us recall some basic notions and terminology we will use. We present: (i) the syntax of (a variant of) the guarded commands [7] which are used for defining concurrent programs, (ii) some basic notions of group theory which are required for defining symmetric concurrent programs, (iii) the syntax and the semantics of the Computation Tree Logic, and (iv) the syntax and the semantics of Answer Set Programming, which is the framework we use for our synthesis method.

Guarded commands. In our variant of the guarded commands we consider two basic sets: (i) variables, $v$ in $V a r$, each ranging over a finite domain $D_{v}$, and (ii) guards, $g$ in Guard, of the form: $g::=$ true $\mid$ false $|v=d| \neg g \mid g_{1} \wedge g_{2}$, with $v \in \operatorname{Var}$ and $d \in D_{v}$. We also have the following derived sets whose definitions are mutually recursive: (iii) commands, $c$ in Command, of the form: 
$c::=$ skip $|v:=d| c_{1} ; c_{2} \mid$ if $g c$ fi $\mid$ do $g c$ od, where ';' denotes the sequential composition of commands, and (iv) guarded commands, gc in GCommand, of the form: $g c::=g \rightarrow c \mid g c_{1} \llbracket g c_{2}$, where '『' denotes the parallel composition of guarded commands.

The execution of if $g c_{1}\|\ldots\| g c_{n} \mathrm{fi}$ is performed as follows: one of the guarded commands $g \rightarrow c$ in $\left\{g c_{1}, \ldots, g c_{n}\right\}$ whose guard $g$ evaluates to true is chosen, then $c$ is executed; otherwise, if no guard in $\left\{g c_{1}, \ldots, g c_{n}\right\}$ evaluates to true then the whole command if ... fi terminates with failure.

The execution of do $g c_{1}[\ldots] g c_{n}$ od is performed as follows: one of the guarded commands $g \rightarrow c$ in $\left\{g c_{1}, \ldots, g c_{n}\right\}$ whose guard $g$ evaluates to true is chosen, then $c$ is executed and the whole command do...od is executed again; otherwise, if no guard in $\left\{g c_{1}, \ldots, g c_{n}\right\}$ evaluates to true then the execution proceeds with the next command.

Symmetric Groups. A group $G$ is a pair $\langle S, \circ\rangle$, where $S$ is given a set and $\circ$ is a binary operation on $S$ satisfying the following axioms: (i) $\forall x, y \in S . x \circ y \in$ $S$ (closure), (ii) $\forall x, y, z \in S .(x \circ y) \circ z=x \circ(y \circ z)$ (associativity), (iii) $\exists e \in$ $S . \forall x \in S . e \circ x=x \circ e=x$ (identity element), and (iv) $\forall x \in S . \exists y \in S . x \circ y=$ $y \circ x=e$ (inverse element). The order of a group $G$ is the cardinality of $S$. For any $x \in S$, for any $n \geq 0$, we write $x^{n}$ to denote the term $x \circ \ldots \circ x$ with $n$ occurrences of $x$. We stipulate that $x^{0}$ is $e$.

A group $G$ is said to be cyclic iff there exists an element $x \in S$, called a generator, such that $S=\left\{x^{n} \mid n \geq 0\right\}$. We write $G_{x}$ to denote the cyclic group generated by $x$.

We denote by $\operatorname{Perm}(S)$ the set of all permutations (that is, bijections) on the set $S$. $\operatorname{Perm}(S)$ is a group whose operation $\circ$ is function composition and the identity $e$ is the identity permutation, denoted $i d$. The order of a permutation $p$ on a finite set $S$ is the smallest natural number $n$ such that $p^{n}=i d$.

Computation Tree Logic. Computation Tree Logic (CTL) is a propositional branching time temporal logic [5].

Let Elem be a finite set of elementary propositions ranged over by $b$. The syntax of a CTL formula $\varphi$ is as follows:

$$
\varphi::=b\left|\varphi_{1} \wedge \varphi_{2}\right| \neg \varphi|\operatorname{EX} \varphi| \operatorname{EG} \varphi \mid \mathrm{E}\left[\varphi_{1} \mathrm{U} \varphi_{2}\right]
$$

Let us introduce the following abbreviations: (i) $\varphi_{1} \vee \varphi_{2}$ for $\neg\left(\neg \varphi_{1} \wedge \neg \varphi_{2}\right)$, (ii) $\mathrm{EF} \varphi$ for $\mathrm{E}[$ true $\mathrm{U} f]$ (iii) $\mathrm{AG} \varphi$ for $\neg \mathrm{EF} \neg \varphi$, (iv) $\mathrm{AF} \varphi$ for $\neg \mathrm{EG} \neg \varphi$, (v) $\mathrm{A}\left[\varphi_{1} \mathrm{U} \varphi_{2}\right]$ for $\neg \mathrm{E}\left[\neg \varphi_{2} \mathrm{U}\left(\neg \varphi_{1} \wedge \neg \varphi_{2}\right)\right] \wedge \neg \mathrm{EG} \neg \varphi_{2}$, (vi) $\mathrm{AX} \varphi$ for $\neg \mathrm{EX} \neg \varphi$, (vii) $\mathrm{A}\left[\varphi_{1} \mathrm{R} \varphi_{2}\right]$ for $\neg \mathrm{E}\left[\neg \varphi_{1} \mathrm{U} \neg \varphi_{2}\right]$, and (viii) $\mathrm{E}\left[\varphi_{1} \mathrm{R} \varphi_{2}\right]$ for $\neg \mathrm{A}\left[\neg \varphi_{1} \mathrm{U} \neg \varphi_{2}\right]$.

We define the semantics of CTL by giving a Kripke structure $\mathcal{K}=\left\langle S, S_{0}, \lambda, R\right\rangle$, where: (i) $S$ is a finite set of states, (ii) $S_{0} \subseteq S$ is a set of initial states, (iii) $R \subseteq S \times S$ is a total transition relation (thus, $\forall u \in S, \exists v \in S,\langle u, v\rangle \in R$ ), and (iv) $\lambda: S \rightarrow \mathcal{P}($ Elem $)$ is a total, labelling function that assigns to every state $s \in S$ a subset $\lambda(s)$ of the set Elem.

For reasons of simplicity, when the set of the initial states is a singleton $\{u\}$, we will feel free to identify $\{u\}$ with $u$.

A path $\pi$ in $\mathcal{K}$ from a state is an infinite sequence $\left\langle s_{0}, s_{1}, \ldots\right\rangle$ of states such that, for all $i \geq 0,\left\langle s_{i}, s_{i+1}\right\rangle \in R$. For $i \geq 0$, we denote by $\pi_{i}$ the $i$-th element 
of $\pi$. The fact that a CTL formula $\varphi$ holds in a state $s$ of a Kripke structure $\mathcal{K}$ will be denoted by $\mathcal{K}, s \vDash \varphi$. For any CTL formula $\varphi$ and state $s$, we define the relation $\mathcal{K}, s \vDash \varphi$ as follows:

$$
\begin{aligned}
& \mathcal{K}, s \vDash b \quad \text { iff } b \in \lambda(s) \\
& \mathcal{K}, s \vDash \neg \varphi \quad \text { iff } \mathcal{K}, s \vDash \varphi \text { does not hold } \\
& \mathcal{K}, s \vDash \varphi_{1} \wedge \varphi_{2} \quad \text { iff } \mathcal{K}, s \vDash \varphi_{1} \text { and } \mathcal{K}, s \vDash \varphi_{2} \\
& \mathcal{K}, s \vDash \operatorname{EX} \varphi \quad \text { iff there exists }\langle s, t\rangle \in R \text { such that } \mathcal{K}, t \vDash \varphi \\
& \mathcal{K}, s \vDash \mathrm{E}\left[\varphi_{1} \cup \varphi_{2}\right] \text { iff there exists a path } \pi=\left\langle s, s_{1}, \ldots\right\rangle \text { in } \mathcal{K} \text { and } i \geq 0 \\
& \mathcal{K}, s \vDash \mathrm{EG} \varphi \quad \text { iff there exists a path } \pi \text { such that } \\
& \pi_{0}=s \text { and for all } i \geq 0, \mathcal{K}, \pi_{i} \vDash \varphi
\end{aligned}
$$

\subsection{Answer Set Programming}

Answer set programming (ASP) is a declarative programming paradigm based on the answer set semantics of logic programs $[10,14]$. We assume the version of ASP with function symbols [3]. Now let us recall some basic definitions of ASP. For those not recalled here we refer to $[3,10,14,15]$. A rule $r$ is an implication of the form:

$a_{1} \vee \ldots \vee a_{k} \leftarrow a_{k+1} \wedge \ldots \wedge a_{m} \wedge \operatorname{not} a_{m+1} \wedge \ldots \wedge \operatorname{not} a_{n}$

where $a_{1}, \ldots, a_{k}, \ldots, a_{n}$ (for $k \geq 0, n \geq k$ ) are atoms and 'not' denotes negation as failure [11]. Given a rule $r$, we define the following sets: head $(r)=\left\{a_{1}, \ldots, a_{k}\right\}$, $\operatorname{pos}(r)=\left\{a_{k+1}, \ldots, a_{m}\right\}$, and neg $(r)=\left\{a_{m+1}, \ldots, a_{n}\right\}$. An integrity constraint is a rule $r$ such that head $(r)=\emptyset$. A logic program is a set of rules. When we write a rule with variables, we actually mean all the ground instances of that rule.

An interpretation $I$ of a program $\Pi$ is a subset of the Herbrand base. The Gelfond-Lifschitz transformation of a program $\Pi$ with respect to an interpretation $I$ is the program $\Pi^{I}=\{\operatorname{head}(r) \leftarrow \operatorname{pos}(r) \mid r \in \Pi \wedge \operatorname{neg}(r) \cap I=\emptyset\}$. An interpretation $M$ is said to be an answer set of $\Pi$ iff $M$ is the least Herbrand model of $\Pi^{M}$. The answer set semantics of $\Pi$ assigns to $\Pi$ a set of answer sets, denoted ans( $\Pi)$. Given an answer set $M \in \operatorname{ans}(\Pi)$ and an atom $a$, we write $M \models a$ to denote that $a \in M$.

\section{Specifying Concurrent Programs}

Let $\mathcal{P}=\left\{P_{1}, \ldots, P_{k}\right\}$ be a finite set of processes. With every process $P_{i} \in \mathcal{P}$ we associate a variable $\mathbf{s}_{i}$, called the local state, ranging over a finite domain $L$, which is the same for all processes. The variable $\mathbf{s}_{i}$ can be tested and modified by $P_{i}$ only. All processes may test and modify also a shared variable $\mathrm{x}$, which ranges over a finite domain $D$.

A concurrent program consists of a finite set $\mathcal{P}$ of processes that are executed in parallel and interact with each other through a communication protocol realized by a set of commands acting on the shared variable $\mathrm{x}$. Here is the formal definition of a concurrent program. 
Definition 1 ( $k$-Process Concurrent Program). Let $L$ be a set of local states and $D$ be a domain of the shared variable $\mathrm{x}$. For any $k>1$, a $k$-process concurrent program $C$ is a command of the form:

$$
C: \quad \mathrm{s}_{1}:=l_{1} ; \ldots ; \mathrm{s}_{k}:=l_{k} ; \mathrm{x}:=d_{0} ; \text { do } P_{1} \rrbracket \ldots \rrbracket P_{k} \text { od }
$$

where $\mathbf{s}_{1}, \ldots, \mathbf{s}_{k}, \mathbf{x} \in \operatorname{Var}, l_{1}, \ldots, l_{k} \in L$, and $d_{0} \in D$.

Every process $P_{i}$ in $P_{1} \rrbracket \ldots \rrbracket P_{k}$ is a guarded command of the form:

$$
P_{i}: \quad \text { true } \rightarrow \text { if } g c_{1} \llbracket \ldots \rrbracket g c_{n} \text { fi }
$$

Every guarded command $g c$ in $g c_{1} \rrbracket \ldots \rrbracket g c_{n}$ is of the form:

$$
\text { gc: } \quad \mathbf{s}_{i}=l \wedge \mathrm{x}=d \rightarrow \mathbf{s}_{i}:=l^{\prime} ; \mathrm{x}:=d^{\prime} ;
$$

where $l, l^{\prime} \in L$ and $d, d^{\prime} \in D$.

We shall use the guarded command $\mathbf{s}_{i}=l \wedge \mathrm{x}=d \rightarrow$ skip as a shorthand for $\mathbf{s}_{i}=l \wedge \mathrm{x}=d \rightarrow \mathbf{s}_{i}:=l ; \mathrm{x}:=d$. The command $\mathbf{s}_{1}:=l_{1} ; \ldots ; \mathbf{s}_{k}:=l_{k} ; \mathrm{x}:=d_{0} ;$ is called initialization of $C$.

Example 1. Let $L$ be the set $\{\mathrm{t}, \mathrm{u}\}$ and $D$ be the set $\{0,1\}$. A 2-process concurrent program $C$ is:

$$
\mathrm{s}_{1}:=\mathrm{t} ; \mathrm{s}_{2}:=\mathrm{t} ; \mathrm{x}:=0 ; \text { do } P_{1} \llbracket P_{2} \text { od }
$$

where $P_{1}$ and $P_{2}$ are defined as follows:

$$
\begin{aligned}
P_{1}: & \text { true } \rightarrow \mathrm{if} \\
& \mathrm{s}_{1}=\mathrm{t} \wedge \mathrm{x}=0 \rightarrow \mathrm{s}_{1}:=\mathrm{u} ; \mathrm{x}:=0 ; \\
\square & \mathrm{s}_{1}=\mathrm{t} \wedge \mathrm{x}=1 \rightarrow \text { skip; } \\
\prod & \mathrm{s}_{1}=\mathrm{u} \wedge \mathrm{x}=0 \rightarrow \mathrm{s}_{1}:=\mathrm{t} ; \mathrm{x}:=1 ; \\
\mathrm{fi} &
\end{aligned}
$$

$$
\begin{aligned}
P_{2}: & \text { true } \rightarrow \text { if } \\
& \mathrm{s}_{2}=\mathrm{t} \wedge \mathrm{x}=1 \rightarrow \mathrm{s}_{2}:=\mathrm{u} ; \mathrm{x}:=1 \\
\square & \mathrm{s}_{2}=\mathrm{t} \wedge \mathrm{x}=0 \rightarrow \text { skip; } \\
\prod & \mathrm{s}_{2}=\mathrm{u} \wedge \mathrm{x}=1 \rightarrow \mathrm{s}_{2}:=\mathrm{t} ; \mathrm{x}:=0 \\
\mathrm{fi} &
\end{aligned}
$$

This program is the familiar program for two processes, each of which either 'thinks' in its noncritical section $\left(\mathbf{s}_{i}=\mathrm{t}\right)$ or 'uses a resource' in its critical section $\left(\mathrm{s}_{i}=\mathrm{u}\right)$. The shared variable $\mathrm{x}$ gives each process its turn to enter the critical section: if $\mathrm{x}=0$, process $P_{1}$ is in its critical section, and if $\mathrm{x}=1$, process $P_{2}$ is in its critical section.

Now we introduce the semantics of concurrent programs by using Kripke structures. We model a state $u$ of a $k$-process concurrent program $C$ by a $(k+1)$-tuple $\left\langle l_{1}, \ldots, l_{k}, d\right\rangle$, where: (i) the first $k$ components are the values of the local state variables $\mathbf{s}_{1}, \ldots, \mathbf{s}_{k}$, and (ii) $d$ is the value of the shared variable $\mathrm{x}$.

Definition 2 (Kripke Structure Associated with a $k$-Process Concurrent Program). Let $C$ be a $k$-process concurrent program of the form

$$
C: \quad \mathrm{s}_{1}:=l_{1} ; \ldots ; \mathrm{s}_{k}:=l_{k} ; \mathrm{x}:=d_{0} ; \text { do } P_{1} \rrbracket \ldots \square P_{k} \text { od }
$$

where the $l_{i}$ 's belong to $L$ and $d_{0}$ belongs to $D$. The Kripke structure $\mathcal{K}$ associated with $C$ is the 4 -tuple $\left\langle S, S_{0}, R, \lambda\right\rangle$, where:

(i) the set $S$ of states is $L^{k} \times D$,

(ii) the set $S_{0}$ of initial states is the singleton $\left\{\left\langle l_{1}, \ldots, l_{k}, d_{0}\right\rangle\right\}$, 
(iii) the set $R \subseteq S \times S$ of transitions

$$
\begin{gathered}
\left\{\langle u, v\rangle \mid i, j \in\{1, \ldots, k\} \wedge \mathbf{s}_{i}=l \wedge \mathrm{x}=d \rightarrow \mathbf{s}_{i}:=l^{\prime} ; \mathrm{x}:=d^{\prime} \text { in } P_{i} \wedge\right. \\
u\left(\mathbf{s}_{i}\right)=l \wedge u(\mathrm{x})=d \wedge v\left(\mathbf{s}_{i}\right)=l^{\prime} \wedge v(\mathrm{x})=d^{\prime} \wedge u \neq v \wedge \\
\left.\forall j \neq i, u\left(\mathbf{s}_{j}\right)=v\left(\mathbf{s}_{j}\right)\right\}
\end{gathered}
$$

where for all states $t \in S$, for all variables $\mathrm{x} \in \operatorname{Var}, t(\mathrm{x})$ denotes the value of the variable $\mathrm{x}$ in $t$, and

(iv) for all states $t$ of the form $\left\langle l_{1}, \ldots, l_{k}, d\right\rangle$, the value $\lambda(t)$ is defined to be $\left\{\mathrm{s}_{1}=l_{1}, \ldots, \mathrm{s}_{k}=l_{k}, \mathrm{x}=d\right\}$.

The set Elem of the elementary propositions is the set $\bigcup_{t \in S} \lambda(t)$.

We make the following assumptions about $k$-process concurrent programs.

(i) Since, by definition, the transition relation $R$ of any Kripke structure is total, we have that every concurrent program $C$ we consider, is nonterminating, in the sense that, in every state there exists a process $P_{i}$ of $C$ and a guarded command $g \rightarrow c$ of $P_{i}$ such that: (i.1) $g$ evaluates to true, and (i.2) $c$ cannot be abbreviated to skip. This assumption restricts the class of concurrent programs we consider.

(ii) Every $k$-process concurrent program consists of deterministic processes, that is, for $i=1, \ldots, k$, in every state, at most one guard of the guarded commands of process $P_{i}$ evaluates to true (a similar assumption is made in [17]).

Note that the usual assumption that every guarded command is executed atomically (in the sense that only one process at a time among the processes of a concurrent program is selected and executed) is taken into account in an implicit way when constructing the transition relation $R$ of the Kripke structure.

Example 2. Given the 2-process symmetric concurrent program $C$ of Example 1, the associated Kripke structure $\left\langle S,\left\{s_{0}\right\}, R, \lambda\right\rangle$ is depicted in Figure 1. We depict it as a graph whose nodes are the states in $S$ and whose edges represent the transitions in $R$. The set $S$ of states includes the four state depicted in Figure 1 and also the states $\langle\mathrm{t}, \mathrm{u}, 0\rangle,\langle\mathrm{u}, \mathrm{t}, 1\rangle,\langle\mathrm{u}, \mathrm{u}, 0\rangle$, and $\langle\mathrm{u}, \mathrm{u}, 1\rangle$, which have not been depicted because they are not reachable from the initial state $\langle t, t, 0\rangle$. Each transition from state $u$ to state $v$ is associated with the guarded command $g \rightarrow c$ whose guard $g$ evaluates to true in $u$. For the labelling function $\lambda$, we have that $\lambda(\langle t, t, 0\rangle)$ is $\left\{\mathbf{s}_{1}=\mathrm{t}, \mathrm{s}_{2}=\mathrm{t}, \mathrm{x}=0\right\}$ and, similarly, for the other states.

Having defined the Kripke structure associated with a given program, now we can define the notion of a program satisfying a given behavioural property.

Definition 3 (Satisfaction relation for a Concurrent Program). Let $C$ be a $k$-process concurrent program, $\mathcal{K}$ be the Kripke structure associated with $C$, $s_{0}$ be the initial state of $\mathcal{K}$, and $\varphi$ be a CTL formula. We say that $C$ satisfies $\varphi$, denoted $C \models \varphi$, iff $\mathcal{K}, s_{0} \models \varphi$.

Example 3. Let us consider the 2-process concurrent program $C$ defined in Example 1. We associate with the local states $t$ (short for 'think') and u (short for 'use') two regions of code, called the noncritical section and the critical section, respectively. We require that the region of code associated with state $u$ should be executed in a mutually exclusive way. This is formalized by the CTL formula 


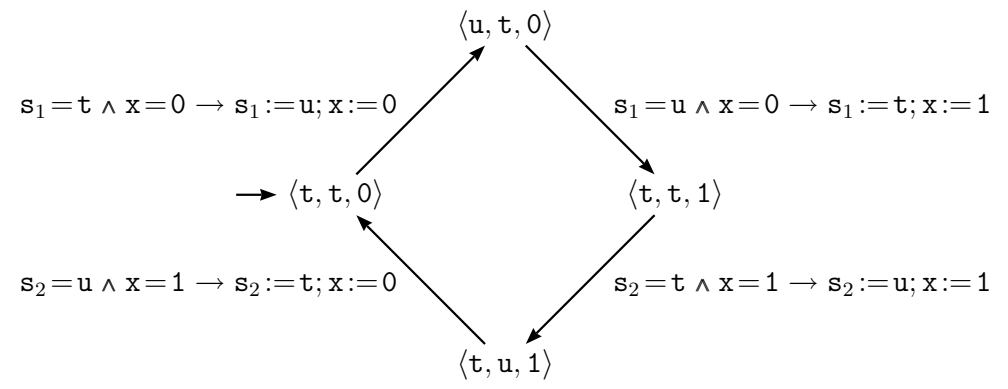

Fig. 1. The transition relation $R$ of the Kripke structure $\mathcal{K}=\left\langle S,\left\{s_{0}\right\}, R, \lambda\right\rangle$ associated with the concurrent program $C$ of Example 1. The initial state $s_{0}$ is $\langle\mathrm{t}, \mathrm{t}, 0\rangle$. The $\operatorname{arcs}$ are labelled by the guarded commands which are responsible for the transition.

$\varphi=\operatorname{def} A G\left[\neg\left(\mathrm{s}_{1}=\mathrm{u} \wedge \mathrm{s}_{2}=\mathrm{u}\right)\right]$, and we have that $C \models \varphi$ holds because for the Kripke structure $\mathcal{K}$ of Example 2 (see Figure 1 ), we have that $\mathcal{K}, s_{0} \models \varphi$ (indeed, there is no path starting from the initial state $s_{0}=\langle\mathrm{t}, \mathrm{t}, 0\rangle$ which leads the system to either the state $\langle\mathrm{u}, \mathrm{u}, 0\rangle$ or the state $\langle\mathrm{u}, \mathrm{u}, 1\rangle)$.

Often, in our setting a $k$-concurrent program consists of symmetric processes, the symmetry being determined by the fact that, for any two processes $P_{i}$ and $P_{j}$, for $i \neq j$, we have that $P_{j}$ can be obtained from $P_{i}$ by permuting the values of the shared variable $\mathrm{x}$ in the guarded commands. Indeed, as shown in Example 1, the guarded commands in $P_{2}$ can be obtained from those in $P_{1}$ by interchanging 0 and 1 . In practice, the property of symmetry is very common in many concurrent programs, and our task is precisely the one of automatically synthesizing symmetric processes. This observation motivates a notion of symmetry which we now introduce by using cyclic groups. A similar approach has been followed for the automated verification of concurrent systems in [8].

Definition 4 ( $k$-Generating Function). Given an integer $k>1$, and a finite domain $D$, we say that $f \in \operatorname{Perm}(D)$ is a k-generating function iff either $f=i d$ or $f$ is a generator of a cyclic group $G_{f}=\left\{i d, f, f^{2}, \ldots, f^{k-1}\right\}$ of order $k$.

Let us introduce the following notation. Given a guarded command $g c$ of the form:

$$
\mathrm{s}_{i}=l \wedge \mathrm{x}=d \rightarrow \mathrm{s}_{i}:=l^{\prime} ; \mathrm{x}:=d^{\prime} ;
$$

and a $k$-generating function $f$, we denote by $f(g c)$ the guarded command:

$$
\mathbf{s}_{(i \bmod k)+1}=l \wedge \mathrm{x}=f(d) \rightarrow \mathbf{s}_{(i \bmod k)+1}:=l^{\prime} ; \mathbf{x}:=f\left(d^{\prime}\right) ;
$$

Definition 5 ( $k$-Process Symmetric Concurrent Program). Given a $k$-generating function $f$, a $k$-process symmetric concurrent program $C$ is a command of the form:

$$
C: \quad \mathrm{s}_{1}:=l_{0} ; \ldots ; \mathrm{s}_{k}:=l_{0} ; \mathrm{x}:=d_{0} ; \text { do } P_{1}[\ldots] P_{k} \text { od }
$$

where, for all processes $P_{i}$, for all guarded commands $g c, g c$ is in $P_{i}$ iff $f(g c)$ is in $P_{(i \bmod k)+1}$. 
Example 4. Let us consider the 2-process concurrent program $C$ of Example 1. The group $\operatorname{Perm}(D)$ of permutations over $D=\{0,1\}$ is made out of the following two permutations only: $f_{1}=\{\langle 0,0\rangle,\langle 1,1\rangle\}$ and $f_{2}=\{\langle 0,1\rangle,\langle 1,0\rangle\}$. The 2 -generating function $f_{2}$ shows that the concurrent program $C$ is symmetric.
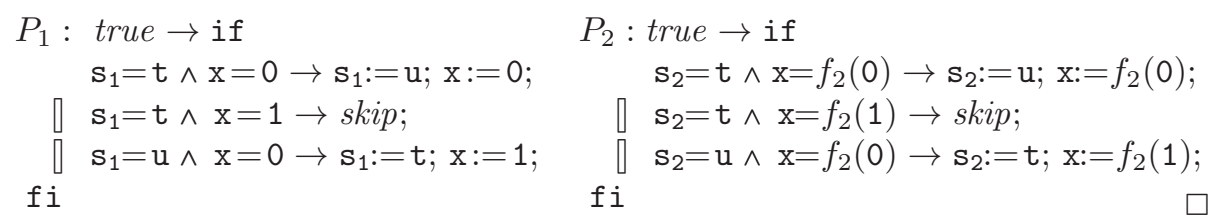

By definition, one can generate a $k$-process symmetric concurrent program $C$ from one of the processes in $C$ by applying the generating function $f$. Moreover, it is often the case that all processes of a given program $C$ also share additional structural properties, besides those determined by $f$. For instance, in the case of Example 4, we have that both process $P_{1}$ and $P_{2}$ may move from the local state $t$ to the local state $u$, or from $t$ to $t$, or from $u$ to $t$. These additional structural properties define a local transition relation $T \subseteq L \times L$ which together with the $k$-generating function $f$, defines a so called symmetric program structure $\Sigma=\langle f, T\rangle$. A pair $\left\langle l, l^{\prime}\right\rangle$ in $T$ will also be denoted by $l \mapsto l^{\prime}$.

Our synthesis problem can be defined as follows.

Definition 6 (Synthesis Problem of a $k$-Process Symmetric Concurrent Program). The synthesis problem of a $k$-process symmetric concurrent program $C$ starting from: (i) a CTL formula $\varphi$, and (ii) a symmetric program structure $\Sigma=\langle f, T\rangle$, where $f$ is a $k$-generating function and $T$ is a local transition relation, consists in finding $C$ such that $C \models \varphi$ holds.

Note that there exists a CTL formula that characterizes the set of initial states. In particular, the initial state $\left\langle l_{1}, \ldots, l_{k}, d_{0}\right\rangle$ can be characterized by the CTL formula $\mathrm{s}_{1}=l_{1} \wedge \ldots \wedge \mathrm{s}_{k}=l_{k} \wedge \mathrm{x}=d_{0}$, where we assume that each conjunct belongs to Elem. However, for reasons of simplicity, we assume that the initial state $s_{0}$ is given to our synthesis procedure as an additional input (see clause 1 of the logic program $\Pi_{\varphi}$ of Definition 7 ).

\section{Synthesising Concurrent Programs}

In this section we present our synthesis procedure based on ASP. We encode the desired behavioural property $\varphi$ of our $k$-process concurrent program to be synthesized as a logic programs $\Pi_{\varphi}$, and the desired structural property $\Sigma$ as a logic programs $\Pi_{\Sigma}$. Programs $\Pi_{\varphi}$ and $\Pi_{\Sigma}$ are defined in the following Definition 7 and 8 , respectively.

Definition 7 (Logic program encoding a behavioural property). Let $\varphi$ be a CTL formula expressing a behavioural property. The logic program $\Pi_{\varphi}$ encoding $\varphi$ is as follows:

1. $\leftarrow \operatorname{not} \operatorname{sat}\left(s_{0}, \varphi\right)$ 


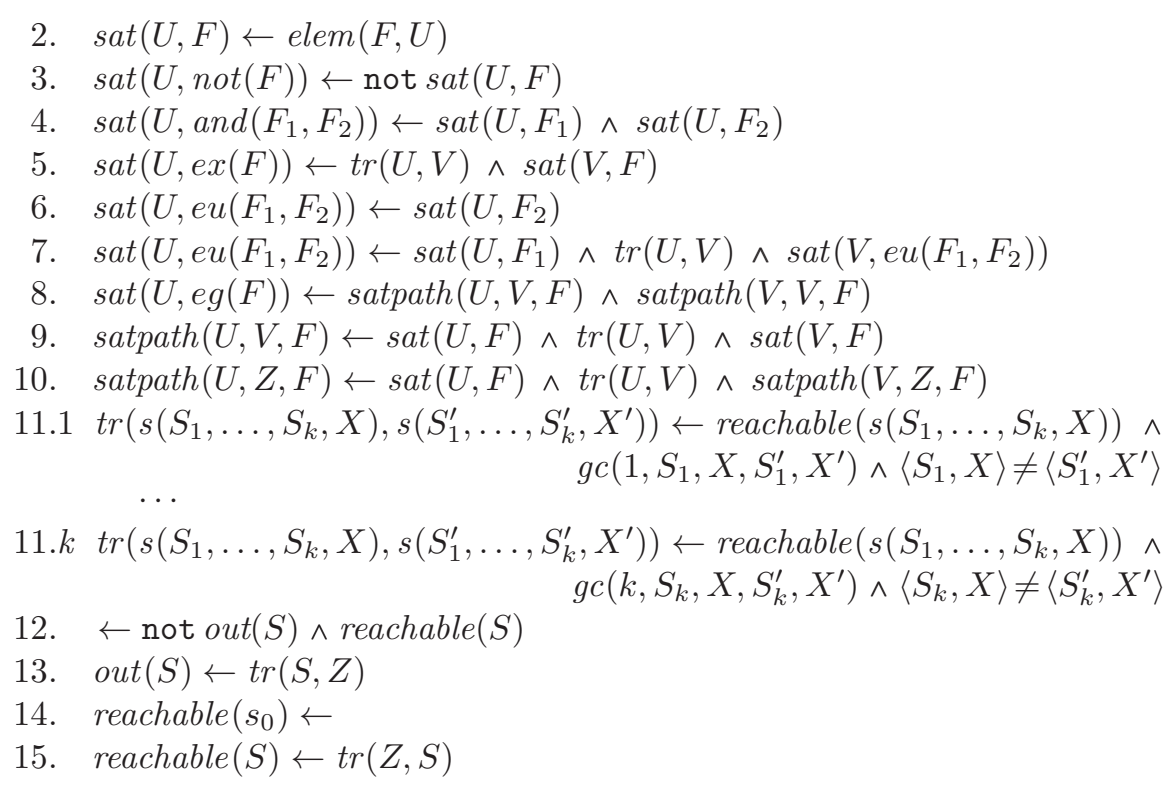

where the predicates are defined as follows: (i) $\operatorname{sat}(U, F)$ holds iff the formula $F$ holds in state $U$, (ii) elem $(b, u)$ holds iff $b \in \lambda(u)$, that is, the elementary proposition $b$ holds in state $u$, (iii) satpath $(U, V, F)$ holds iff there exists a path from state $U$ to state $V$ such that every state in that path satisfies the formula $F$, (iv) $\operatorname{tr}\left(s\left(S_{1}, \ldots, S_{k}, X\right), s\left(S_{1}^{\prime}, \ldots, S_{k}^{\prime}, X^{\prime}\right)\right)$ holds iff the pair of states $\left\langle\left\langle S_{1}, \ldots, S_{k}, X\right\rangle,\left\langle S_{1}^{\prime}, \ldots, S_{k}^{\prime}, X^{\prime}\right\rangle\right\rangle$ belongs to the transition relation $R$ of the Kripke structure associated with the program $C$ to be synthesized, and (v) the predicates out and reachable force the relation $R$ to be total (in particular, out $(S)$ holds iff from state $S$ there is an outgoing edge, and reachable $(S)$ holds iff there is a path from the initial state $s_{0}$ to state $S$.)

Rule 1 is required for ensuring that $\varphi$ holds in the initial state $s_{0}$ representing the initialization $\mathrm{s}_{1}:=l_{0} ; \ldots ; \mathbf{s}_{k}:=l_{0} ; \mathbf{x}:=d_{0}$ of the $k$-process symmetric concurrent program to be synthesized. Rule 11.i defines the interleaved execution of the guarded commands, that is, for all states $U$ and $V, \operatorname{tr}(U, V)$ holds iff $U$ is a reachable state, and there exists a guarded command $g c$ of process $P_{i}$ whose guard evaluates to true in $U$ and whose execution leads from state $U$ to state $V$.

Definition 8 (Logic program encoding a structural property). Let $L$ be the set of local states and $D$ be the domain of the shared variable. Let $\Sigma=\langle f, T\rangle$ be a symmetric program structure of a $k$-process symmetric concurrent program. The logic program $\Pi_{\Sigma}$ is defined as follows:

$$
\begin{aligned}
& 1.1 \bigvee_{\left\langle S^{\prime}, X^{\prime}\right\rangle \in \operatorname{Next}\left(\left\langle S_{1}, X\right\rangle\right)} g c\left(1, S_{1}, X, S^{\prime}, X^{\prime}\right) \leftarrow \operatorname{reachable}\left(S_{1}, S_{2}, \ldots, S_{k}, X\right) \\
& 1.2 \quad \leftarrow g c\left(1, S, X, S^{\prime}, X^{\prime}\right) \wedge g c\left(1, S, X, S^{\prime \prime}, X^{\prime \prime}\right) \wedge\left\langle S^{\prime}, X^{\prime}\right\rangle \neq\left\langle S^{\prime \prime}, X^{\prime \prime}\right\rangle \\
& 2.1 \quad g c\left(2, S, f(X), S^{\prime}, f\left(X^{\prime}\right)\right) \leftarrow g c\left(1, S, X, S^{\prime}, X^{\prime}\right) \\
& 2.2 \quad \leftarrow g c\left(2, S, X, S^{\prime}, X^{\prime}\right) \wedge \operatorname{not} p s(2, S, X)
\end{aligned}
$$




$$
\begin{array}{ll}
2.3 & p s\left(2, S_{2}, X\right) \leftarrow \text { reachable }\left(S_{1}, S_{2}, \ldots, S_{k}, X\right) \\
& \ldots \\
k .1 & g c\left(k, S, f(X), S^{\prime}, f\left(X^{\prime}\right)\right) \leftarrow g c\left(k-1, S, X, S^{\prime}, X^{\prime}\right) \\
k .2 & \leftarrow g c\left(k, S, X, S^{\prime}, X^{\prime}\right) \wedge \operatorname{not} p s(k, S, X) \\
k .3 & p s\left(k, S_{k}, X\right) \leftarrow \operatorname{reachable}\left(S_{1}, S_{2}, \ldots, S_{k}, X\right)
\end{array}
$$

where: (i) $g c\left(i, S, X, S^{\prime}, X^{\prime}\right)$ holds iff $\mathrm{s}_{\mathrm{i}}=l \wedge \mathrm{x}=d \rightarrow \mathrm{s}_{\mathrm{i}}:=l^{\prime} ; \mathrm{x}:=d^{\prime}$ is in $P_{i}$, (ii) $f$ is a $k$-generating function, (iii) $p s(i, S, X)$ holds iff there exists a reachable state of the form $\left\langle S_{1}, \ldots, S_{i-1}, S, S_{i+1}, \ldots, S_{k}, X\right\rangle$, and (iv) for all $l \in L, d \in D$, $\operatorname{Next}(l, d)=\left\{\left\langle l^{\prime}, d^{\prime}\right\rangle \mid l \mapsto l^{\prime} \in T \wedge d^{\prime} \in D\right\}$.

Rules 1.1 and 1.2 generate a set of guarded commands for process $P_{1}$. The disjunction in the head of Rule 1.1 is over all possible guarded commands that $P_{1}$ may execute. The set of those guarded commands is defined using the sets $N e x t(l, d)$, one for each $l \in L$ and $d \in D$. The integrity constraint 1.2 enforces the generation of a set of guarded commands in which any two guards of the guarded commands in $P_{1}$ are mutually exclusive (recall that we consider only deterministic processes).

For $j=2, \ldots, k$, Rules $j .1, j .2$ and $j .3$ realize Definition 5 . We use Rule $j .1$ to derive a guarded command in $P_{j}$ from a guarded command of the process $P_{j-1}$. Rule $j .2$ ensures that for every guarded command $g \rightarrow c$ derived by $j .1$, there exists a reachable state $U$ such that in $U$ the guard $g$ evaluates to true.

Now we present a theorem establishing the correctness of our synthesis procedure. It relates the $k$-process symmetric concurrent programs satisfying $\varphi$ with the answer sets of the logic program $\Pi_{\varphi} \cup \Pi_{\Sigma}$. Obviously, the correctness of the synthesis procedure implies also the correctness of the programs $\Pi_{\varphi}$ and $\Pi_{\Sigma}$ encoding the behavioural properties and the structural properties, as specified in Definition 7 and 8, respectively.

Theorem 1 (Correctness of Synthesis). Let $\Pi=\Pi_{\varphi} \cup \Pi_{\Sigma}$ be the logic program obtained, as specified by Definitions 7 and 8, from: (i) a CTL formula $\varphi$ and (ii) a symmetric program structure $\Sigma=\langle f, T\rangle$. Then,

$$
\left(\mathrm{s}_{1}:=l_{0} ; \ldots ; \mathrm{s}_{\mathrm{k}}:=l_{0} ; \mathrm{x}:=d_{0} ; \text { do } P_{1} \rrbracket \ldots \rrbracket P_{k} \text { od }\right) \models \varphi
$$

iff there exists an answer set $M$ in ans $(\Pi)$ such that

$\forall i \in\{1, \ldots, k\}, \forall l, l^{\prime} \in L, \forall d, d^{\prime} \in D$,

$$
\left(\mathbf{s}_{i}=l \wedge \mathrm{x}=d \rightarrow \mathbf{s}_{i}:=l^{\prime} ; \mathrm{x}:=d^{\prime}\right) \text { is in } P_{i} \quad \text { iff } M \models \operatorname{gc}\left(i, l, d, l^{\prime}, d^{\prime}\right) \text {. }
$$

\section{Experimental Results}

In this section we present some experimental results obtained by applying our synthesis procedure to mutual exclusion protocols. All experiments have been performed on an Intel Core 2 Duo E7300 2.66GHz under the Linux operating system.

The first synthesis we did is the one of a simple program, called 2-mutex-1, for two processes enjoying the mutual exclusion property only, and then we progressively increased the number of properties that the synthesized program should 
satisfy (see Table 1). In that table the program $k$-mutex- $p$ denotes a synthesized program for $k$ processes satisfying $p$ behavioural properties. For instance, program 2-mutex-4 is the synthesized program that works for 2 processes and enjoys the four behavioural properties: (i) $M E$ (mutual exclusion), (ii) $S F$ (starvation freedom), (iii) $B O$ (bounded overtaking), and (iv) $M R$ (maximal reactivity), defined by CTL formulas as follows.

(i) Mutual Exclusion, that is, it is not the case that process $P_{i}$ is in its critical section $\left(\mathbf{s}_{i}=\mathrm{u}\right)$, and process $P_{j}$ is in its critical section $\left(\mathbf{s}_{j}=\mathrm{u}\right)$ at the same time: for all $i, j$ in $\{1, \ldots, k\}$, with $i \neq j$,

$$
\mathrm{AG} \neg\left(\mathrm{s}_{i}=\mathrm{u} \wedge \mathrm{s}_{j}=\mathrm{u}\right)
$$

(ii) Starvation Freedom, that is, if a process is waiting to enter the critical section $\left(\mathbf{s}_{i}=\mathrm{w}\right)$, then after a finite amount of time, process $P_{i}$ will execute its critical section $\left(\mathrm{s}_{i}=\mathrm{u}\right)$ : for all $i$ in $\{1, \ldots, k\}$,

$$
\mathrm{AG}\left(\mathrm{s}_{i}=\mathrm{w} \rightarrow \mathrm{AF} \mathrm{s}_{i}=\mathrm{u}\right)
$$

(iii) Bounded Overtaking, that is, while process $P_{i}$ is in its waiting section, any other process $P_{j}$ exits from its critical section at most once: for all $i, j$ in $\{1, \ldots, k\}$,

$$
\mathrm{AG}\left(\left(\mathrm{s}_{i}=\mathrm{w} \wedge \mathrm{s}_{j}=\mathrm{u}\right) \rightarrow \mathrm{AF}\left(\mathrm{s}_{j}=\mathrm{t} \wedge \mathrm{A}\left[\neg\left(\mathrm{s}_{j}=\mathrm{u}\right) \mathrm{U} \mathrm{s}_{i}=\mathrm{u}\right]\right)\right)(B O)
$$

(iv) Maximal Reactivity, that is, if process $P_{i}$ is waiting to execute the critical section and all other processes are executing their noncritical sections, then in the next state $P_{i}$ will enter its critical section: for all $i$ in $\{1, \ldots, k\}$,

$$
\mathrm{AG}\left(\left(\mathrm{s}_{i}=\mathrm{w} \wedge \bigwedge_{j \in\{1, \ldots, k\} \backslash\{i\}} \mathrm{s}_{j}=\mathrm{t}\right) \rightarrow \mathrm{EX} \mathbf{s}_{i}=\mathrm{u}\right)
$$

In our synthesis experiments we have made the following choices for $s_{0}, L, D$, $f$, and $T$.

The initial state $s_{0}$ is $\langle t, t, 0\rangle$ and $\langle t, t, t, 0\rangle$ for the 2- and 3-process symmetric concurrent programs, respectively.

The set $L$ of the local states for the variables $\mathbf{s}_{i}$ 's is $\{\mathrm{t}, \mathrm{w}, \mathrm{u}\}$, where $\mathrm{t}$ represents the noncritical section, w represents the waiting section, and $\mathrm{u}$ represents the critical section.

The domain $D$ of the shared variable $\mathrm{x}$ is a finite set of natural numbers whose cardinality $|D|$ depends on: (i) the number $k$ of the processes to be synthesized, and (ii) the properties that the concurrent program should satisfy. The value of $|D|$ is not known a priori, and we guess it at the beginning of our synthesis task. If the synthesis fails, we increase the value of $|D|$, hoping for a successful synthesis with a larger value of $|D|$.

The $k$-generating function $f$ is chosen among the following ones: (i) $i d$ is the identity function, (ii) $f_{1}=\{\langle 0,1\rangle,\langle 1,0\rangle\}$, (iii) $f_{2}=\{\langle 0,1\rangle,\langle 1,0\rangle,\langle 2,2\rangle\}$, and (iv) $f_{3}=\{\langle 0,1\rangle,\langle 1,2\rangle,\langle 2,0\rangle\}$.

The local transition relation $T$ is $\{\mathrm{t} \mapsto \mathrm{w}, \mathrm{w} \mapsto \mathrm{w}, \mathrm{w} \mapsto \mathrm{u}, \mathrm{u} \mapsto \mathrm{t}\}$. The pair $\mathrm{t} \mapsto \mathrm{w}$ denotes that, once the noncritical section has been executed, a process enters the waiting section. The pairs $\mathrm{w} \mapsto \mathrm{w}$ and $\mathrm{w} \mapsto \mathrm{u}$ denote that a process may repeat (possibly an unbounded number of times) the execution of its waiting section and then may enter its critical section. The pair $u \mapsto t$ denotes that, once the critical section has been executed, a process enters its noncritical section. 


\begin{tabular}{|l|l|c|c|r|r|}
\hline Program & Satisfied Properties & $|D|$ & $f$ & $\mid$ ans $(\Pi) \mid$ & Time \\
\hline \hline 2-mutex-1 & $M E$ & 2 & $i d$ & 6 & 0.07 \\
\hline 2-mutex-1 & $M E$ & 2 & $f_{1}$ & 7 & 0.70 \\
\hline 2-mutex-2 & $M E, S F$ & 2 & $f_{1}$ & 3 & 0.71 \\
\hline 2-mutex-3 & $M E, S F, B O$ & 2 & $f_{1}$ & 3 & 1.44 \\
\hline 2-mutex-4 & $M E, S F, B O, M R$ & 3 & $f_{2}$ & 2 & 11.7 \\
\hline \hline 3-mutex-1 & $M E$ & 2 & $i d$ & 5 & 0.95 \\
\hline 3-mutex-1 & $M E$ & 2 & $f_{1}$ & 10 & 0.87 \\
\hline 3-mutex-2 & $M E, S F$ & 3 & $f_{3}$ & 8 & 152 \\
\hline 3-mutex-3 & $M E, S F, B O$ & 3 & $f_{3}$ & 8 & 1700 \\
\hline
\end{tabular}

Table 1. Column named Program gives the names of the synthesized programs. $k$-mutex- $p$ denotes the mutual exclusion program for $k$ processes and $p$ behavioural properties that are indicated in the column named Satisfied Properties. $M E, S F, B O$ and $M R$ stand for 'mutual exclusion', 'starvation freedom', 'bounded overtaking', and 'maximal reactivity', respectively. Column named $|D|$ gives the cardinality of the domain of the shared variable $\mathrm{x}$. Column named $f$ gives the $k$-generating functions (they are defined in the text). Column named $\mid$ ans $(\Pi) \mid$ gives the cardinality of ans $(\Pi)$, that is, the number of answer sets of program $\Pi=\Pi_{\varphi} \cup \Pi_{\Sigma}$. In column named Time we indicate the times (in seconds) taken for the synthesis using the smodels [20].

In Figures 2 and 3 we present the syntax and the semantics of the synthesized program, called 2-mutex-4, for the 2-process mutual exclusion problem described in Example 3. (Program 2-mutex-4 is essentially the same as the Peterson algorithm [18], but it uses a single shared variable.)

\section{Related Work and Concluding Remarks}

Two well known, early works on synthesis of concurrent programs were those by Emerson and Clark [6] and Manna and Wolper [16].

In [6] Emerson and Clark introduce the notion of a synchronization skeleton as an abstraction of the actual processes in concurrent programs. They synthesize programs for a shared-memory model of execution by extracting the synchronization skeletons from the models of CTL specifications using a tableau-based decision procedure for the satisfiability of CTL formulas. This extraction procedure is not completely mechanized.

Similarly to [6] in [16] Manna and Wolper present a method for synthesizing synchronization instructions for processes in a message-passing model of execution from a Propositional Temporal Logic (PTL) using a tableau-based decision procedure for the satisfiability of PTL formulas. The instructions synthesized by their method are written as Communicating Sequential Processes [13].

In [19] Piterman, Pnueli, and Sa'ar consider the problem of the design of digital circuits from Linear Temporal Logic (LTL) specifications and give an 

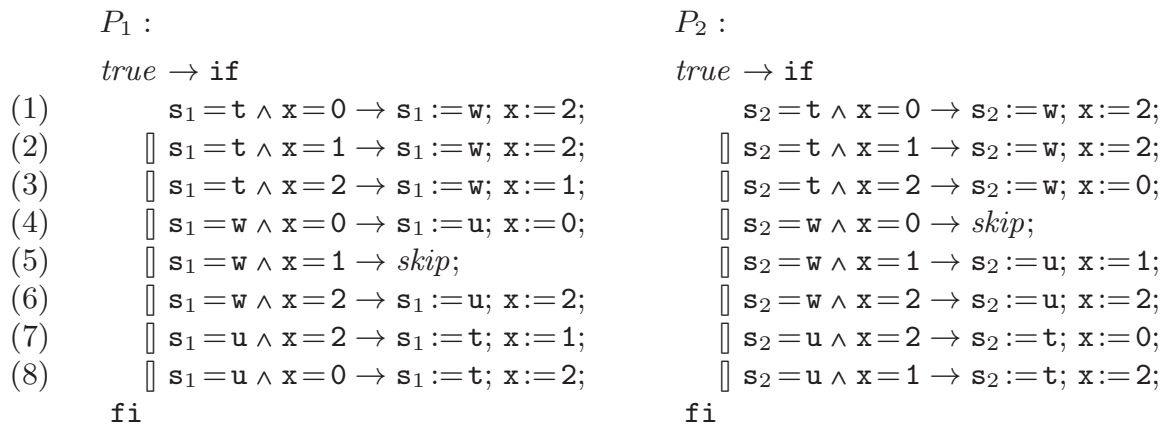

Fig. 2. The two synthesized processes $P_{1}$ and $P_{2}$ of the program 2-mutex-4: $\mathrm{s}_{1}:=\mathrm{t}$; $\mathrm{s}_{2}:=\mathrm{t} ; \mathrm{x}:=0 ;$ do $P_{1} \llbracket P_{2}$ od. It enjoys the following properties: mutual exclusion, starvation freedom, bounded overtaking, and maximal reactivity.

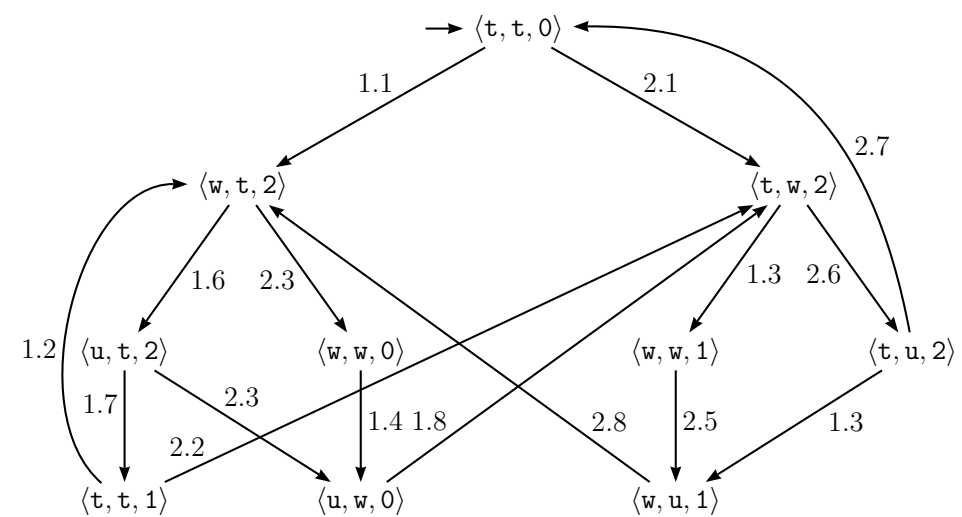

Fig. 3. The transition relation of the Kripke structure associated with the 2-process concurrent program 2-mutex-4. The initial state is $\langle\mathrm{t}, \mathrm{t}, 0\rangle$. For $i=1,2$, an arc labelled $i . n$ indicates that the guarded command $n$ of process $P_{i}$ is responsible for that transition.

$O\left(N^{3}\right)$ algorithm to construct an automaton satisfying a formula of a particular class of LTL specifications.

We closely follow the approaches of [6] and [16]. In particular we synthesize concurrent processes that communicate with each other by means of shared variables starting from CTL specifications. The programs we synthesize are written as guarded commands [7].

In order to reduce the search space of our synthesis problem, we have used a notion of symmetric concurrent programs which is similar to the one which was introduced in $[1,8]$ to overcome the state explosion problem. Our notion of symmetry is formalized using group theory, similarly to what has been done in [8] for model checking.

Similarly to Attie and Emerson [2], we also propose a method for the synthesis task and we separate the behavioural properties from the structural properties. 
However, in our approach the structural properties, such as symmetry, are represented in the symmetric program structures, rather than an automata based formalism.

We have implemented our synthesis method in Answer Set Programming (ASP). One advantage of our method over $[1,6,16]$ is its generality: besides temporal properties, we can specify structural properties, such as the above mentioned symmetry, and our ASP program will automatically synthesize concurrent programs satisfying the desired properties without the need for ad hoc algorithms.

To the best of our knowledge, there is only one paper by Heymans, Nieuwenborgh and Vermeir [12] who use Answer Set Programming for the synthesis of concurrent programs. They have extended the ASP paradigm by adding preferences among models and they have developed an answer set system, called OLPS. Using OLPS they perform the synthesis of concurrent programs following the approach proposed in [6]. The synthesis method is not completely automatic and, in particular, the shared variables are manually introduced during the extraction of the synchronization skeleton. We do not require any extension of the ASP paradigm, we use the by now standard ASP systems, such as DLV [9] and smodels [20], and every steps of our synthesis procedure is fully automatic.

As future work we plan to explore various techniques for reducing the search space of the synthesis procedure and, thus, we hope to synthesize protocols for a larger number of processes and more complex properties to be guaranteed. Among these techniques we envisage to apply those used in compositional model checking [4].

\section{References}

1. P. C. Attie and E. A. Emerson. Synthesis of Concurrent Programs with Many Similar Processes ACM Trans. on Program. Lang. and Syst., 51-115, 1998.

2. P. C. Attie and E. A. Emerson. Synthesis of Concurrent Programs for an Atomic Read/Write Model of Computation. ACM Trans. Program. Lang. Syst., 187-242, 2001.

3. F. Calimeri, S. Cozza, G. Ianni and N. Leone. Enhancing ASP by Functions: Decidable Classes and Implementation Techniques. Proceedings of the 24-th AAAI Conference on Artificial Intelligence 2010, 1666-1670, 2010.

4. E. M. Clarke Jr., D. E. Long, and K. L. McMillan. Compositional model checking. Logic in Computer Science, LICS '89, Proceedings, IEEE Computer Society, 353$362,1989$.

5. E. M. Clarke Jr., O. Grumber and D. A. Peled. Model Checking. The MIT Press, 1999.

6. E. M. Clarke and E. A. Emerson. Design and Synthesis of Synchronization Skeletons Using Branching Time Temporal Logic. Workshop on Logic of Programs, London, UK, Springer-Verlag, 52-71, 1982.

7. E. W. Dijkstra. A Discipline of Programming. Prentice-Hall, 1976.

8. E. A. Emerson and A. P. Sistla. Symmetry and Model Checking. Formal Methods in System Design: 9, 1-2, 105-131, 1996. 
9. N. Leone, G. Pfeifer, W. Faber, T. Eiter, G. Gottlob, S. Perri and F. Scarcello. The DLV system for knowledge representation and reasoning ACM Trans. Comput. Logic: 7, 499-562, 2006.

http://www.dlvsystem.com/dlvsystem/index.php/DLV

10. M. Gelfond and V. Lifschitz. The Stable Model Semantics For Logic Programming. Proc. of the Fifth Intern. Conf. and Symp. on Logic Programming, Seattle, MIT Press, 1070-1080, 1988.

11. M. Gelfond and V. Lifschitz. Classical Negation in Logic Programs and Disjunctive Databases. New Generation Computing: 9, 365-385, 1991.

12. S. Heymans, D. Van Nieuwenborgh and D. Vermeir. Synthesis from Temporal Specifications using Preferred Answer Set Programming. Lecture Notes in Computer Science no. 3701, Springer, 280-294, 2005.

13. C.A.R. Hoare. Communicating Sequential Processes. Prentice-Hall, 1985.

14. V. Lifschitz. Answer Set Programming and Plan Generation. Artificial Intelligence no. 138, 39-54, 2002.

15. V. Lifschitz. What Is Answer Set Programming? Proceedings of the AAAI Conference on Artificial Intelligence, MIT Press, 1594-1597, 2008.

16. Z. Manna and P. Wolper: Synthesis of Communicating Processes from Temporal Logic Specifications. ACM Trans. Program. Lang. Syst., 68-93, 1984.

17. Z. Manna and A. Pnueli. The Temporal Logic of Reactive Systems: Specification. Springer-Verlag, 1991.

18. G. L. Peterson. Myths about the mutual exclusion problem. Information Processing Letters, 12(3):115-116, 1981.

19. N. Piterman, A. Pnueli and Y. Sa'ar. Synthesis of Reactive(1) Designs. Lecture Notes in Computer Science no. 3855, Springer, 364-380, 2006.

20. T. Syrjänen and I. Niemelä. The Smodels System. Lecture Notes in Computer Science no. 2173, Springer, 434-438, 2001.

http://www.tcs.hut.fi/Software/smodels/ 